\title{
La dispersión urbana y los costes temporales de la movilidad: el caso de Matadepera, Región Metropolitana de Barcelona
}

\author{
Giulio Mezzetti \\ Anna Badia \\ Meritxell Gisbert \\ Universitat Autònoma de Barcelona. Departament de Geografia \\ mezzetti92@gmail.com \\ anna.badia@uab.cat \\ meritxell.gisbert@uab.cat
}

Recibido: noviembre de 2019

Aceptado: julio de 2020

Publicado: octubre de 2020

\section{Resumen}

El consumo excesivo de suelo, la pérdida de biodiversidad, la descohesión social y la pérdida de identidad, la privatización del paisaje o los costes temporales de la movilidad son algunos de los impactos de la dispersión urbana. Este artículo se centra en el análisis de la movilidad cotidiana en un municipio de la Región Metropolitana de Barcelona, Matadepera. Se analizan los costes temporales de la movilidad — en coche y a pie— utilizando dos metodologías distintas del análisis de redes: 1) matriz de costes OD desde cada portal a los servicios básicos y 2) cálculo de áreas de servicio a partir de distintos puntos de interés. El objetivo es cuantificar y cartografiar la accesibilidad de la población para explicar el alto nivel de dependencia del transporte motorizado privado. Los resultados demuestran cómo la dispersión urbana, y más especialmente en zonas de relieve pronunciado, afecta directamente la modalidad de transporte y dificulta el uso de alternativas de movilidad más sostenibles.

Palabras clave: urbanización dispersa; accesibilidad urbana; costes temporales; análisis de redes 
Resum. La dispersió urbana i els costos temporals de la mobilitat: el cas de Matadepera, Regió Metropolitana de Barcelona

El consum excessiu de sòl, la pèrdua de biodiversitat, la descohesió social i la pèrdua d'identitat, la privatització del paisatge o els costos temporals de la mobilitat són alguns dels impactes de la dispersió urbana. Aquest article se centra en l'anàlisi de la mobilitat quotidiana en un municipi de la Regió Metropolitana de Barcelona, Matadepera. S'analitzen els costos temporals de la mobilitat —en cotxe i a peu- utilitzant dues metodologies diferents de l'anàlisi de xarxes: 1) matriu de costos OD des de cada portal als serveis bàsics, i 2) càlcul d'àrees de servei a partir de punts d'interès. L'objectiu és fer una representació i cartografiar l'accessibilitat de la població per explicar el nivell de dependència del transport motoritzat privat. Els resultats demostren com la dispersió urbana, i molt especialment en zones de relleu pronunciat, afecta directament la modalitat de transport i dificulta l'ús d'alternatives de mobilitat més sostenibles.

Paraules clau: urbanització dispersa; accessibilitat urbana; costos temporals; anàlisi de xarxes

Résumé. Dispersion urbaine et coûts temporels de la mobilité : Le cas de Matadepera, Région Métropolitaine de Barcelone

Une consommation excessive de terres, une perte de biodiversité, un manque de cohésion sociale et une perte d'identité, la privatisation du paysage ou des coûts temporaires de la mobilité sont quelques-uns des impacts de la dispersion urbaine. Cet article porte sur l'analyse de la mobilité quotidienne dans une municipalité de la Région Métropolitaine de Barcelone, Matadepera. Les coûts temporaires de la mobilité sont analysés, pour les déplacements en voiture et à pied, en utilisant deux méthodes différentes de l'analyse des réseaux, 1) la matrice des coûts OD depuis chaque portail jusqu'aux services de base et 2) le calcul des zones de service à partir de différents points d'intérêt. L'objectif est de quantifier et de cartographier l'accessibilité de la population afin d'expliquer le niveau élevé de dépendance au transport privé motorisé. Les résultats démontrent que la dispersion urbaine, et plus particulièrement dans les zones au relief prononcé, affecte directement le mode de transport et entrave l'utilisation d'alternatives de mobilité plus durables.

Mots-clés: urbanisation dispersée; accessibilité urbaine; coûts temporaires; analyse de réseau

Abstract. Urban sprawl and temporary mobility costs: The case of Matadepera, Barcelona Metropolitan Region

Excessive land consumption, loss of biodiversity, social lack of cohesion and loss of identity, privatization of the landscape or temporary mobility costs are some of the impacts of urban sprawl. This paper focuses on the analysis of daily mobility in a municipality of the Metropolitan Region of Barcelona, Matadepera. The temporary costs of mobility, by car and on foot, are analyzed using two different network analysis methods: 1) OD cost matrix from each housing portal to basic services and 2) calculation of service areas from different points of interest. The objective is to quantify and map the population's accessibility in order to explain the high level of dependence on private transport. The results demonstrate how urban dispersion, especially in high slope areas, directly affects the mode of transport and hinders the use of more sustainable mobility alternatives.

Keywords: urban sprawl; urban accessibility; temporary costs; network analysis 


\section{Sumario}
1. Introducción
4. Análisis y discusión de los resultados
2. Área de estudio
5. Conclusiones
3. Datos y metodología
Referencias bibliográficas

\section{Introducción}

La preocupación existente por el fenómeno de dispersión urbana se ponía en evidencia con la publicación del informe de la Agencia Europea del Medio Ambiente Urban sprawl in Europe - The ignored challenge (EEA, 2006). Diez años más tarde, aparece un nuevo informe actualizado, con información mucho más precisa y que tiene como objetivo controlar la expansión urbana (EEA, 2016). Según este documento, la población humana tenderá a crecer con toda probabilidad, lo que comportará que la producción de alimentos y las necesidades de desarrollo urbano compitan entre sí por el suelo. Según una noticia de las Naciones Unidas, en 2018 más de la mitad de la población mundial (55\%) vivía en zonas urbanas (en 1930 era del 30\%) (ONU, 2018). El binomio sociedad y naturaleza propia del mundo rural, donde la cubierta forestal era el medio de subsistencia (Cussó et al., 2005), va desapareciendo, lo que supone una pérdida de aquellas cubiertas ligadas a una realidad económica vinculada a las actividades primarias tradicionales.

El crecimiento de la industria automovilística fue un factor determinante en la «reducción» de las distancias entre áreas rurales y urbanas, factor que comportó un progresivo desplazamiento (sobre todo en los años sesenta) desde los centros de las ciudades a las áreas periurbanas (Banzo, 2005). Frecuentemente, se hace referencia a este modelo de urbanización como el prototipo americano de ciudad, y se asocia el modelo europeo de ciudad al de ciudad compacta, con una estructura histórica compleja. Aunque el fenómeno de dispersión urbana se generalizó en Estados Unidos a partir de la década de los años cincuenta del siglo XX, coincidiendo con el auge del coche, puede afirmarse que este fenómeno afecta a toda Europa, aunque empiece más tarde (a finales de los años setenta) y con menor propagación e impacto (Schneider y Woodcock, 2008; Salvati et al., 2013; González, 2017).

Genéricamente, la dispersión urbana se ha definido como aquel suelo que se ha ido convirtiendo progresivamente en uso urbano y que se opone al modelo de ciudad compacta (Samonà, 1968; Brueckner y Fansler, 1983; Lowry, 1988; Indovina, 1990; Hasse y Lathrop, 2003). La EEA define la dispersión urbana como el modelo físico de expansión de baja densidad de grandes áreas urbanas, bajo las condiciones de mercado, que tiene lugar en los alrededores de zonas agrícolas (EEA, 2006). Galster et al. (2001) centran la definición en la disminución de ocho valores importantes en el desarrollo urbano: densidad, continuidad, concentración, agrupación, centralidad, nuclearidad, mezcla de usos y proximidad. Muñiz y García-López (2013) proponen la siguiente definición: proceso de 
expansión urbana que implica la descentralización de la población y un territorio desconcentrado, poco denso, discontinuo y desestructurado.

Los impactos ambientales, sociales y económicos que la urbanización dispersa tiene sobre el territorio han sido debatidos extensamente en la literatura. Algunos de los más destacados son los siguientes: el consumo excesivo de suelo (Hasse y Lathrop, 2003; Eigenbrod et al., 2011); la fragmentación de los hábitats (Radeloff et al., 2005; Lambin et al., 2001); la pérdida de biodiversidad (Palomino y Carrascal, 2006; Turbé et al., 2010); la potencial dispersión de las especies exóticas de los jardines (Honnay et al., 1999; Guirado, 2005); la privatización de espacios abiertos (Muñoz, 2003; Bruegmann, 2005); el incremento de la movilidad a causa del uso del transporte privado (Martínez-Alier y Roca, 2001; Travisi, et al., 2010); los elevados consumos de agua (Domene y Saurí, 2006; March y Saurí, 2010); la desestructuración del territorio, sin una ordenación urbanística ni una distribución de los servicios bien definida (Muñiz y García-López; 2013); los elevados costes municipales del mantenimiento de las infraestructuras urbanas y de servicios (Hortas-Rico y Solé-Ollé, 2010).

La ocupación extensiva de suelo artificial que supone la dispersión urbana dificulta la planificación y la gestión del territorio. El acceso a las compras cotidianas y a los servicios más básicos, como la educación o la sanidad, resulta un problema. Pero también los servicios de agua, alcantarillado, electricidad, redes de banda ancha y la misma red viaria son un coste añadido, no solo su implantación, sino también su mantenimiento. Además, el diseño de una red de transporte público es mucho más complejo y con costes económicos mucho más elevados, lo que convierte el coche como la alternativa más viable.

Así pues, el transporte privado es un elemento clave para la movilidad en la vida cotidiana de las personas que habitan en zonas de baja densidad residencial, al permitirles ahorrar tiempo en el desplazamiento (Palma y Rochat, 2000; Gardner y Abraham, 2007; Miralles-Guasch et al., 2014). En el estudio sobre la movilidad cotidiana en Barcelona de Marquet y Miralles-Guash (2014a) o en el de dinámicas de proximidad de los mismos autores (2014b), se considera que un desplazamiento de proximidad es aquel que tiene una distancia a pie menor a $650 \mathrm{~m}$ y en el que se destinan menos de 10 minutos. La encuesta de movilidad de la Región Metropolitana de Barcelona del IERMB (2006) expone que el 38,6\% de los desplazamientos no motorizados son de más de 10 minutos (en los que una persona a paso normal puede desplazarse entre $800 \mathrm{y}$ 1.200 metros, dependiendo de las condiciones de cada individuo). De todas formas, la encuesta también afirma que en los municipios pequeños de menos de 10.000 habitantes (Matadepera no llega a esta cifra) las distancias realizadas a pie son menores. Un estudio con niños y adolescentes considera que la distancia máxima óptima a recorrer a pie por parte de los niños para desplazarse a la escuela se sitúa entre los $675 \mathrm{~m}$ y los $1.250 \mathrm{~m}$, según se trate de zonas rurales o urbanas, respectivamente. Por parte de los adolescentes, esta distancia se sitúa entre los 1.350 m y los 1.550 m (Rodríguez-López et al., 2017). Teóricamente, la movilidad en bicicleta podría ser el medio de transporte más eficiente, pero la disponibilidad de carriles bici está lejos de ser óptimo. 
El análisis de redes se ha utilizado habitualmente para evaluar la movilidad y la accesibilidad en las ciudades. De hecho, existen diferentes estudios sobre este tema, como el análisis sobre los puntos focales de la red viaria en Wuhan (China), para evaluar la distribución del desplazamiento de las personas utilizando las trayectorias de los taxis (Zhou et al., 2015); el estudio sobre la accesibilidad del transporte público en Londres de Ford et al. (2015), o el de Adís Abeba (Etiopía) de Gebeyehu y Takano (2008). Todos estos estudios se han realizado con los datos de población agregados, pero existen algunos que analizan la movilidad y la accesibilidad con datos de población desagregados, ya sea por sexo (Pérez et al., 2017) o por grupos de edad (Marquet y MirallesGuasch, 2014b; Páez et al., 2011). A pesar de esto, la diagnosis de los inconvenientes de la urbanización dispersa en cuanto a la movilidad a partir de redes es bastante inexplorada, y menos aún la utilización de esta metodología de análisis de redes. Uno de los estudios más próximos a la línea de investigación que se plantean en este artículo es el de Sevtsuk y Mekonnen (2012) y Sevtsuk et al. (2016), que combinan fórmulas matemáticas y herramientas de análisis de redes para calcular cinco tipos de centralidades en redes urbanas (calles y edificios urbanos): alcance, índice de gravedad, intermediación, cercanía y rectitud. De todas maneras, el estudio no analizaba la movilidad en zonas de urbanización dispersa, sino que se aplicó en grandes ciudades de Australia y Estados Unidos, y con la finalidad de analizar la forma urbana y la viabilidad para los peatones de las manzanas o bloques.

El objetivo general de este artículo es analizar los costes temporales que comporta, en términos de movilidad cotidiana, la actual estructura urbana a los ciudadanos residentes en el municipio de Matadepera. Concretamente, se pretende evaluar el coste en tiempo que la población necesita para llegar a los servicios básicos en función de su lugar de residencia, y cuál es el área de influencia de cada uno de estos servicios. El análisis se realiza a partir de diferentes medios de desplazamiento — en coche y a pie- y considerando algunos datos estadísticos de la población. Se trata de un trabajo que tiene una doble aportación. Por un lado, la principal novedad es la metodología utilizada. Se trata de la aplicación de dos módulos distintos de la extensión Network Analyst de ArcGis: 1) matriz de costes OD desde cada portal a los servicios básicos y 2) cálculo de áreas de servicio a partir de distintos puntos de interés. Por el otro, esta metodología se aplica en un caso de estudio especial del que no existen precedentes, un municipio con una urbanización dispersa. El objetivo es cuantificar y cartografiar la accesibilidad de la población para explicar el alto nivel de dependencia del transporte privado. En este artículo no se tendrán en cuenta las excepcionalidades de la población en cuanto a la accesibilidad contemplada en la Orden VIV/561/2010, de 1 de febrero.

Con este objetivo, se formulan las siguientes preguntas de investigación: ¿cuál es el coste en tiempo desde cada vivienda hasta un servicio determinado?, ¿cuál es el área de influencia en tiempo desde un servicio determinado? Para responder estas preguntas, se plantea la siguiente hipótesis de trabajo: la movilidad de la población residente en Matadepera se sustenta básicamente 
en el uso del transporte privado por la misma configuración estructural del territorio.

\section{2. Área de estudio}

El área de estudio es un municipio de la Región Metropolitana de Barcelona (figura 1), con una superficie de $24 \mathrm{~km}^{2}$. Se trata de una circunscripción que es un ejemplo de estructura residencial clásica de baja densidad. Con los años ha perdido su carácter rural a favor del residencial, y ha pasado de un mosaico agroforestal a uno residencial forestal (Badia y Valldeperas, 2015). En enero de 2019, la población total era de 9.505 habitantes (Ajuntament de Matadepera). La distribución territorial de la población es bastante homogénea por toda el área urbana, compuesta por el Casco Antiguo y 18 urbanizaciones o barrios. Si bien es cierto que el Casco Antiguo cuenta con más residentes (2.210 habitantes, el 23,1\% del total), existen varias urbanizaciones alejadas del centro urbano, con poblaciones que oscilan entre los 500 y los 1.000 residentes.

La población se distribuye de forma uniforme entre hombres y mujeres (4.763 hombres y 4.742 mujeres). Es interesante resaltar como la población es relativamente joven respecto a la media catalana. Los menores de 20 años

Figura 1. Área de estudio y distribución de la población por barrios

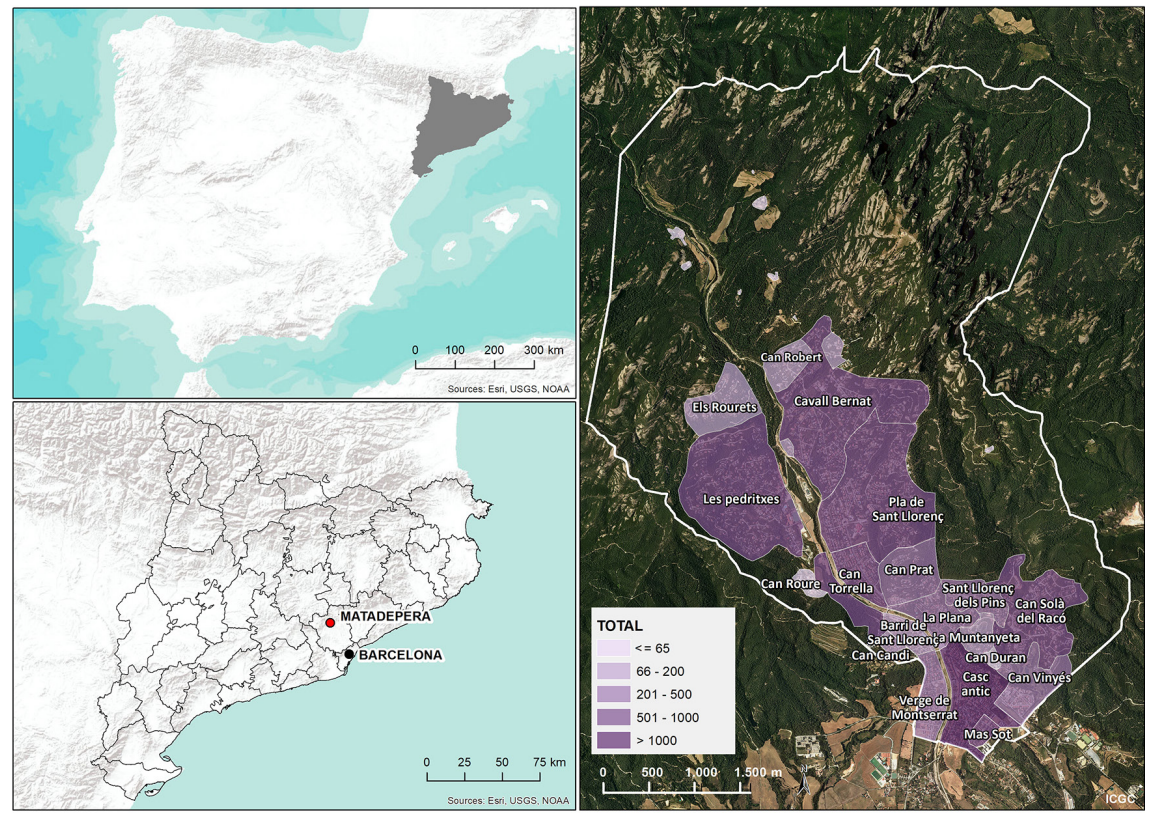

Fuente: elaboración propia. 
son el 26,3\%, y solo un 10,92\% son mayores de 70 años. Matadepera presenta una estructura de edades relativamente joven y poco envejecida; la población menor de 14 años es mayor que la población de más de 65 años, a diferencia de las cifras para el conjunto de Cataluña (Idescat, n. d.). De los 9.505 residentes, 1.118 son niños entre los 0 y los 10 años $(11,76 \%), 1.385$ son adolescentes entre los 11 y los 20 años $(14,57 \%)$ y 1.038 son mayores de 70 años. Se pone énfasis en estos grupos de edades por la incidencia que tienen en la movilidad. Los primeros dependen de un adulto para acceder a sus actividades diarias (escuelas, zonas deportivas, parques, etcétera), mientras que en el segundo caso y a medida que envejecen la movilidad en vehículo privado motorizado les resulta más costosa.

Matadepera se encuentra a una distancia de aproximadamente $30 \mathrm{~km}$ de la ciudad de Barcelona y a unos 3-5 km de dos grandes ciudades de la Región Metropolitana de Barcelona, Sabadell y Terrassa (con poco más de 200.000 habitantes cada una). Por un lado, las características principales del municipio son su elevada superficie dentro del parque natural de Sant Llorenç del Munt i la Serra de l'Obac (aproximadamente el 60\%) y, por el otro, el crecimiento sostenido de la población durante las últimas cuatro décadas, que se ha producido gracias a la construcción de viviendas unifamiliares, lo que ha dado lugar a una estructura horizontal de la urbanización. El área urbana de Matadepera se compone de urbanizaciones de baja densidad, con un predominio de viviendas unifamiliares, mayoritariamente aisladas (Muñoz, 2003), insertadas en un entorno básicamente forestal. El 70\% del parque de viviendas supera los $120 \mathrm{~m}^{2}$, cuando el peso de estas viviendas en el conjunto del parque en Catalunya apenas alcanza el 12\% (Idescat, n. d.). Esto es indicativo del nivel socioeconómico de la población de Matadepera. La renta familiar disponible por habitante en 2016 en Matadepera era de 26.400 euros, mientras que la de Catalunya era de 17.000 euros (Idescat, n. d.). Es de esperar que los hábitos de movilidad de una población con un nivel económico superior a la media y que vive en una zona dispersa se basen en el uso del transporte privado.

Según el Mapa Urbanístico de Catalunya (MUC, n. d.) de 2018, el uso del suelo urbano es esencialmente residencial. De las 456,5 ha que suman los usos residenciales, el 94,3\% corresponde a residencial aislado. En cambio, la superficie del municipio ocupada por actividades económicas es irrelevante, apenas 10 ha (0,4\% del total del municipio). Así, no es de extrañar que mayoritariamente la población deba desplazarse fuera del municipio para trabajar.

La preeminencia del vehículo privado, la morfología urbana y el diseño de un espacio público totalmente sesgado para el uso del vehículo privado dificultan el cambio en los modos de desplazamiento en el municipio (Cebollada et al., 2020). Además, la falta de un trasporte público eficiente obliga a que los desplazamientos se lleven a cabo con medios de trasporte privados. Los distintos servicios y actividades (comercios, centros sanitarios) están concentrados preferentemente en el centro, y el resto de territorio queda como uso residencial, lo que genera barrios dormitorio. De hecho, las únicas actividades que se pueden encontrar en las urbanizaciones son restaurantes, zonas deportivas 
y escuelas. Así pues, para las compras más habituales, la población debe desplazarse al centro o a otras ciudades. Dadas las distancias y la organización del transporte público, los residentes utilizan preferiblemente el coche. Finalmente, cabe destacar que en Matadepera a cada habitante le corresponden 12,6 m de calle asfaltada, mientras que en las ciudades más próximas de Terrassa y Sabadell esta cifra se divide por más de cinco, 2,1 m y 2,2 m, respectivamente (calculado a partir de la base cartográfica de redes de transporte del IGN y de la población de 2018 del Idescat). Los costes de mantenimiento y de abastecimiento de servicios son cinco veces mayores en una población de poco más de 9.000 habitantes que en las poblaciones vecinas de Terrassa y Sabadell, con poco más 200.000 habitantes cada una.

\section{Datos y metodología}

No existen referentes del uso de la metodología aplicada al análisis de los costes temporales de la movilidad en zonas de baja densidad residencial. La complejidad de esta metodología se basa en el uso de distintas fuentes de datos y su actualización y preparación para aplicar el análisis de redes.

\subsection{Fuentes de datos}

Los datos utilizados para el análisis proceden de seis fuentes de información distintas.

Límite municipal (ICGC, n. d.): se ha extraído el límite municipal de Matadepera en formato Shapefile y a escala 1:5.000.

Redes de transporte viario, viales urbanos e interurbanos (IGN, n. d.): esta capa en formato Shapefile contiene todas las vías urbanas e interurbanas de la provincia de Barcelona. Se ha extraído el viario correspondiente al municipio de Matadepera, que se ha actualizado con la ayuda de Google Street View (noviembre de 2018), fotointerpretación (junio de 2018) y trabajo de campo para revisar algunos de los tramos que se prestaban a confusión (junio de 2019). Además, se ha actualizado el campo relativo al revestimiento de las calles (1.- pavimentado, 2.- tratamiento superficial y 3.- sin tratamiento superficial), el de los sentidos (1.- único y 2.- doble) y el de tipo de vehículo que puede circular (100.- peatón, 110.- peatón + bici y 111.- peatón + bici + vehículo). A partir de esta información corregida, y después de revisar que los arcos de calle sigan el sentido de circulación correspondiente, se ha generado una columna llamada Oneway para definir las direcciones de las calles (N: dirección prohibida, TF: dirección prohibida en la dirección del arco, FT: dirección prohibida en dirección contraria al arco y 00: no posee dirección prohibida, es una calle de sentido doble). Esta última información es muy importante para aplicar correctamente el análisis de redes con vehículo privado motorizado. Finalmente, se añaden los límites de velocidad para cada vial. Esta información, no disponible en ninguna institución, se ha obtenido a partir de Google Street View (noviembre de 2018) y con trabajo de campo 
para revisar algunos tramos que no era posible identificar con claridad (junio de 2019). En total, se han definido 4 velocidades: $50 \mathrm{~km} / \mathrm{h}$ (carreteras interurbanas), $40 \mathrm{~km} / \mathrm{h}$ (zonas urbanas - avenidas), $30 \mathrm{~km} / \mathrm{h}$ (zonas urbanas o calles sin asfalto) y $20 \mathrm{~km} / \mathrm{h}$ (zonas urbanas próximas a escuelas y zonas deportivas).

Parcelas catastrales (Sede Electrónica del Catastro, n. d.): estos datos incluyen toda la estructura parcelaria de Matadepera, también en formato Shapefile y a escala 1:5.000. La Dirección General del Catastro permite la descarga en dos ficheros separados, uno para las parcelas urbanas y otro para las rurales. Esta capa se ha utilizado para el control de calidad de la capa de portales.

Portales (Instituto Geográfico Nacional): la información contenida en esta capa, en formato Shapefile, es de gran importancia para el análisis de redes. Los portales, en formato de puntos (origen), son necesarios para evaluar el coste temporal de desplazamiento hasta los puntos de interés (destino).

Puntos de interés (Diputació de Barcelona, n. d.): esta capa, en formato Shapefile, se ha obtenido del Sistema d'Informació Territorial de la Diputació de Barcelona (Sitmun, n. d.) y contiene todos los servicios de Matadepera (supermercados, farmacias, escuelas, restaurantes, paradas de autobús, etcétera). Mediante el uso de Google Street View (noviembre de 2018) y con trabajo de campo (junio de 2019), se ha actualizado la capa (se han eliminado los servicios no existentes, se han añadido los nuevos y se ha cambiado el tipo de actividad). Además, también se le han añadido dos campos: uno que especifica el tipo de servicio (p. e., Frutería) y otro que especifica el sector (p. e., Actividad comercial). Estos puntos (destino) son necesarios para evaluar el coste temporal de desplazamiento desde las viviendas (origen) o para calcular el área de influencia.

Reductores de velocidad (elaboración propia): son todos aquellos dispositivos elevados que obligan a un vehículo a reducir la velocidad. Este tipo de información no existía en ninguna institución. Para localizar estos dispositivos, se utilizaron tres fuentes: fotointerpretación (junio de 2018), Google Street View (noviembre de 2018) y trabajo de campo (junio de 2019). En total, entre badenes y pasos de peatones elevados, se han localizado 44 reductores de velocidad en la red viaria de Matadepera. Disponer de esta información es útil para añadir costes temporales en el cálculo del análisis de redes para vehículos privados motorizados.

LiDAR (Institut Cartogràfic i Geològic de Catalunya): estos datos, 12 hojas en total del año 2016, tienen una densidad mínima de 0,5 puntos $/ \mathrm{m}^{2}$ y se distribuyen en formato LAS comprimido (LAZ) (ICGC, n. d.). Cada punto LiDAR contiene la siguiente información asociada: intensidad, número de devoluciones, clasificación de puntos, borde de la línea de vuelo, RGB, tiempo del GPS, ángulo de escaneo y dirección de escaneo. El ICGC suministra las hojas LiDAR ya clasificadas de la siguiente forma: 0 (never classified), 1 (not assigned), 2 (ground), 3 (low vegetation), 4 (medium vegetation), 5 (high vegetation), 6 (building), 7 (low point), 8 (model key points), 9 (water), 10 (rail), 11 (road), 12 (reserved), 13 (cable protector), 14 (cable conductor), 15 (transmission tower), 16 (cable structure connector), 17 (bridge platform), 18 (high noise), 19-63 (reserved) y 64-255 (defined by the user). A partir de los datos LiDAR, 
se ha creado el Modelo Digital del Terreno (MDT) de Matadepera, del que se han obtenido las capas derivadas de pendiente y de orientación.

Población de 2019 (Ajuntament de Matadepera, n. d.): estos datos alfanuméricos contienen los habitantes agrupados por sexo, por grupos de edad y el total, a escala de barrio. Estos datos son básicos para estudiar el contexto sociodemográfico y detectar así las áreas que por edad de los residentes tienen mayor problemática en relación con los costes temporales de desplazamiento.

\subsection{Metodología}

En el apartado de fuentes de datos, se han especificado algunas de las funciones de geoprocesamiento previas al análisis de redes. La capa principal es la red de transporte viario que ha requerido ser geoprocesada para, por un lado, actualizar los viales y sus características y, por el otro, añadir la información necesaria para ejecutar el análisis de redes. El proceso para analizar los costes de la movilidad cotidiana urbana en Matadepera se detalla en el flujo de trabajo de la figura 2.

Los costes de la movilidad se han cuantificado y mapificado atendiendo a dos necesidades: a) la caracterización de la red viaria según los parámetros físicos de pendiente y orientación y $b$ ) el análisis del coste origen-destino y el análisis de áreas de influencia.

Figura 2. Flujo de trabajo para estudiar los costes de la movilidad cotidiana urbana

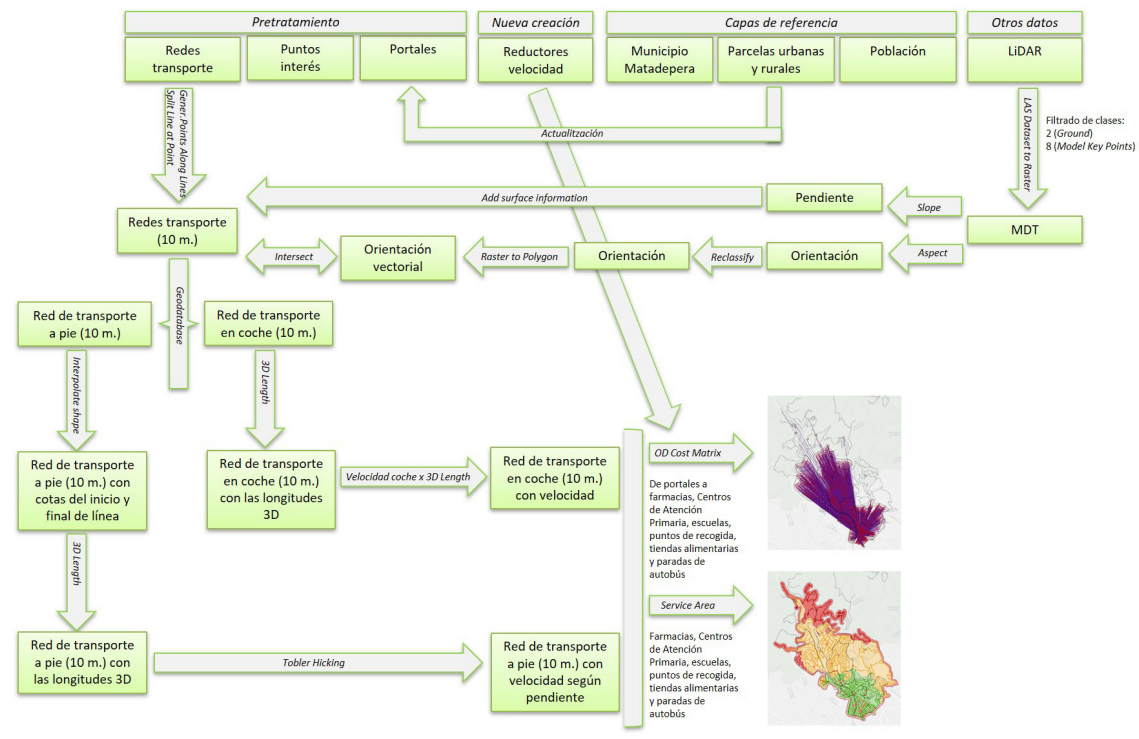

Fuente: elaboración propia. 


\section{a) Caracterización de la red de transporte viario}

La caracterización de la red viaria, según pendiente y orientación, se ha realizado con el objetivo de identificar aquellos viales que suponen o pueden suponer alguna restricción para la movilidad. Un vial orientado al norte y con una pendiente pronunciada puede ser un inconveniente en una situación meteorológica adversa (heladas). Además, una pendiente pronunciada afecta directamente al coste de la movilidad a pie. La pendiente asociada al vial es un atributo importante en la ejecución del coste de la movilidad temporal a pie. Las capas de pendientes y orientación proceden del MDT. Este MDT se ha creado a partir de las 12 hojas LiDAR descargadas del ICGC. Los pasos realizados hasta la obtención final de los modelos son: a) conversión del formato comprimido .laz a formato .las (con el software libre LAStools), para poder manipularlas con ArcGis; $b$ ) creación de un paquete Las Dataset de ArcGis con todos los ficheros . las. Los datos LiDAR tienen diferentes códigos de clasificación y retornos, por lo que antes de proceder a crear el MDT se tienen que filtrar por la clase 2 (ground) y la 8 (model key points) y por todos los retornos (all returns); c) generación del MDT con una resolución de $0,5 \mathrm{~m}$; d) generación de los modelos de orientaciones y pendientes.

La asignación de estas variables a cada tramo requiere disponer de la capa de viales fragmentada para tener información más precisa (no es lo mismo la media de pendiente u orientación de un tramo de calle de $50 \mathrm{~m}$ que la media en un tramo de $10 \mathrm{~m}$ ). Por ello, los viales se han partido en tramos de $10 \mathrm{~m}$ y se les ha asignado la media de pendiente u orientación a partir de las capas obtenidas del MDT. Con esta información es posible cuantificar y mapificar el porcentaje de longitud de los viales en cada intervalo de pendiente $(0-2 \%$ -plano, 2-5\% -suave, 5-10\% -moderado, 10-20\% -elevado y > 20\% -escarpado) y el porcentaje de los viales en cada clase de orientación (norte, este, sur, oeste y plano).

\section{b) Análisis de redes: coste origen-destino y áreas de influencia}

$\mathrm{El}$ análisis de redes para estudiar los costes de la movilidad cotidiana urbana se ha realizado con la herramienta Network Analyst de ArcGis, que dispone de un amplio abanico de funcionalidades para trabajar con redes de transporte. En el caso que nos ocupa, se ha trabajado con dos de ellas: áreas de servicio, para encontrar todas las calles accesibles desde un punto de origen, en un tiempo o distancia especificados, y matriz de coste OD, para encontrar y calcular las rutas de menor coste desde varios puntos de origen hasta varios puntos de destino.

Se han seleccionado siete puntos de interés básicos (farmacias, centro de atención primaria, escuelas, punto de evacuación, comercio de alimentación, paradas de autobús y estación de bomberos). En todos los casos se ha realizado el análisis de matriz OD para elaborar una tabla con el coste en tiempo de cada portal a cada servicio, y el área de influencia de cada servicio. En el caso de la estación de bomberos, solo se ha ejecutado el área de servicio.

A partir de la capa de redes de transporte, se han generado dos datasets - colección de clases de entidad relacionadas que comparten un sistema 
de coordenadas común (ArcGis, n. d.) - , uno para calcular el coste a pie y otro para calcular el coste en coche. La preparación de los datasets ha consistido en:

- Dataset en coche: en primer lugar, se ha calculado el campo longitud en 3D, para tener más precisión. A partir del campo de las velocidades asignadas a cada tramo en función de la tipología, se ha calculado el coste en minutos de cada tramo en dos fases: 1) conversión del campo que contiene la velocidad en función de la tipología en $\mathrm{km} / \mathrm{hr}$ a $\mathrm{m} / \mathrm{min}\left[(\mathrm{km} / \mathrm{hr})^{*} 1000 / 60\right]$ y 2) cálculo del coste de cada tramo [longitud del tramo/m_min].

- Dataset a pie: la pendiente tiene un efecto real en la movilidad, por lo que ha sido necesario conocer la cota inicial y final de cada tramo y así saber si la calle asciende o desciende. Además de estos campos, es necesario disponer de la longitud plana (campo por defecto de la base cartográfica) y de la longitud en 3D (calculado como en el caso del coste en coche). Disponer del primer campo es necesario para el cálculo de velocidades, mientras que el segundo es necesario para el cálculo de los costes. Finalmente, se ha utilizado la función de Tobler Hicking para encontrar la velocidad relativa a la pendiente según el ángulo de inclinación. Para ello, se han creado dos campos: F_T_Slope (from-to) y T_F_Slope (to-from). Mediante el lenguaje Python de la calculadora de campos, se han aplicado las siguientes formulas a un campo y otro:

$$
\begin{aligned}
& F_{-} T_{-} S l o p e=6 * \text { match. } \exp \left(-35 * \text {, math. fabs }\left(\frac{! \mathrm{Z}_{\mathrm{fin} !}-! \mathrm{Z}_{\mathrm{ini} !}}{! \text { Shape }_{\mathrm{Lengh!}}+0.05}\right)\right)
\end{aligned}
$$

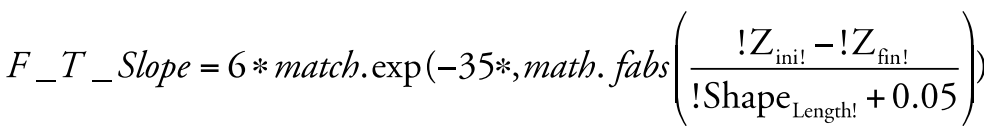

Observando los resultados, es posible ver que en las pendientes positivas la velocidad va disminuyendo a medida que se avanza en el sentido de la pendiente, mientras que en los valores de pendiente negativa la velocidad va aumentando hasta el punto de que empieza a decrecer (con menor intensidad a como baja la velocidad cuesta arriba).

Las velocidades asignadas a cada tramo permiten crear dos campos donde calcular los costes temporales:

$$
\begin{gathered}
F_{-} T_{-} \text {Minutos }=\left(\frac{! \text { Length }_{3 \mathrm{D}}}{1000}\right) * \frac{60}{! F_{\text {TSlope }} !} \\
T_{-} F_{-} \text {Minutos }=\left(\frac{! \text { Length }_{3 \mathrm{D}} !}{1000}\right) * \frac{60}{! T_{-} F_{-} \text {Slope } !}
\end{gathered}
$$


Con los datasets preparados, se ha creado la red para ejecutar análisis con las características especificadas en la tabla 1.

Con este proceso, que genera dos capas (arcos y nodos), se ha generado la red a partir de la que se ha ejecutado la matriz de coste OD y el área de servicio, una para cada punto de interés (tabla 2). El primer proceso permite encontrar la ruta más rápida (en minutos) desde un punto de origen a un punto de destino teniendo en cuenta los sentidos de las calles (en coche), la pendiente (a pie) y las impedancias (en coche).

El segundo proceso permite encontrar las calles que están dentro de un tiempo especificado y, por lo tanto, conocer el área de influencia que queda abastecido (tabla 3).

La ejecución de este proceso ha permitido analizar los costes de la movilidad cotidiana urbana en Matadepera de los servicios básicos y principales.

Tabla 1. Características de los Networks Datasets

\begin{tabular}{lll}
\hline Características & Coche & Pie \\
\hline Giros & Permitidos & Cualquier vértice \\
Conectividades & Permitidos & Cualquier vértice \\
Modelización elevaciones & No & No \\
Sentidos de calles & Sí & F_T_Minutos y T_F_Minutos \\
Coste temporal & Coste (minutos) & Long. (metros) \\
Longitud & Long. (metros) & A pie \\
Modalidad de transporte & Coche & Sí \\
Permiso giros en U & Sí, solo en calles sin salida & Los campos del tipo de tramo y de nombre de tramo son los \\
Nombre de la red & que definen la red. & \\
& \multicolumn{2}{l}{} \\
\hline
\end{tabular}

Fuente: elaboración propia.

Tabla 2. Características de las matrices de coste

\begin{tabular}{lll}
\hline Matriz de coste & Coche & \multicolumn{1}{c}{ A pie } \\
\hline Punto de origen & Portales & \multicolumn{1}{c}{ Portales } \\
Punto de destino & $\begin{array}{l}\text { Farmacias, Centro de Atención Primaria, escuelas, } \\
\text { puntos de recogida, comercio de alimentación y para- } \\
\text { das de autobús. En el análisis de coste de la estación } \\
\text { de bomberos, la lógica marca que el punto de destino } \\
\text { sean los portales }\end{array}$ \\
Coste añadido (impedimentos) & $\begin{array}{l}\text { Sí, } 5 \text { segundos por cada } \\
\text { reductor de velocidad }\end{array}$ & No \\
Vueltas U & No & De un punto de origen al punto de destino más cercano \\
Número de puntos de origen a destino
\end{tabular}

Fuente: elaboración propia. 
Tabla 3. Características de las áreas de servicio

\begin{tabular}{lll}
\hline Área de servicio & Coche & A pie \\
\hline Punto de origen & $\begin{array}{l}\text { Farmacias, Centro de Atención Primaria, escuelas, puntos de } \\
\text { evacuación, comercio de alimentación y paradas de autobús }\end{array}$ \\
\hline Coste añadido (impedimentos) & $\begin{array}{l}\text { Sí, } 5 \text { segundos por cada } \\
\text { reductor de velocidad }\end{array}$ & No \\
\hline Dirección del cálculo & $\begin{array}{l}\text { En dirección al punto de origen. En el análisis de la estación de } \\
\text { bomberos, la lógica marca que la dirección del cálculo sea desde } \\
\text { la estación de bomberos }\end{array}$ \\
\hline Vueltas U & No & Sí \\
\hline Irrupciones de tiempo & $\begin{array}{l}\text { Cada 5 minutos hasta los 15 } \\
\text { minutos }\end{array}$ & $\begin{array}{l}\text { Cada 5 minutos hasta los 30 } \\
\text { minutos y, después, cada 15 } \\
\text { minutos }\end{array}$ \\
\hline
\end{tabular}

Fuente: elaboración propia.

\section{Análisis y discusión de los resultados}

La principal aportación de este trabajo se centra en los resultados obtenidos a partir del análisis de redes. De todas formas, la mapificación (figura 3) y la cuantificación (tabla 4) de las características físicas (pendiente y orientación)

Figura 3. Pendiente y orientación de las calles de Matadepera por tramos

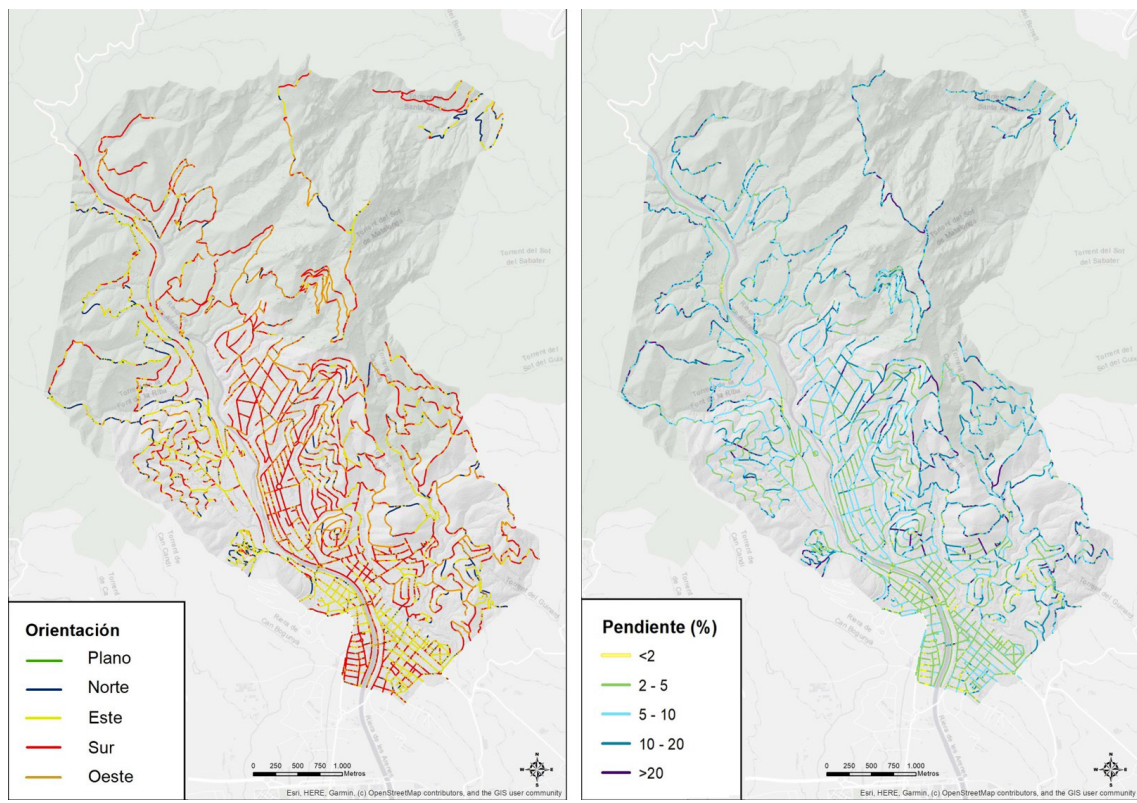

Fuente: elaboración propia a partir de la base cartográfica de redes de transporte del IGN y del MDT, obtenida a partir de datos LiDAR del ICGC. 
Tabla 4. Características de los tramos de las calles en relación con la pendiente y la orientación

\begin{tabular}{lccc}
\hline Pendiente (\%) & \% tramos calle & Orientación & $\%$ tramos calle \\
\hline$<2$ & 1,8 & Plano & 0,0 \\
$2-5$ & 32,4 & Norte & 12,1 \\
$5-10$ & 36,7 & Este & 26,5 \\
$10-20$ & 23,6 & Sur & 39,7 \\
$>=20$ & 5,4 & Oeste & 21,7 \\
\hline
\end{tabular}

Fuente: elaboración propia a partir de la base cartográfica de redes de transporte del IGN y del MDT, obtenida a partir de datos LiDAR del ICGC.

de la red viaria, a partir de la asignación de la pendiente y la orientación a cada uno de los tramos de la red viaria, permiten evaluar otros costes relacionados con la movilidad en situaciones climáticas o ambientales adversas (nevadas, heladas, ventadas, inundaciones, incendios, etcétera). Disponer de esta información es imprescindible en la toma de decisiones y en la priorización de la intervención en situaciones críticas.

A las distancias propias de la dispersión urbana hay que añadirle la pendiente pronunciada que dificulta la movilidad. Más del $25 \%$ de las calles tienen una pendiente por encima del 10\%, y un 36,7\% de las calles tienen una pendiente entre el $5 \%$ y el 10\%. En cuanto a la orientación de las calles, un 12\% están orientadas al norte y un $21,7 \%$ al oeste, lo que dificulta enormemente la movilidad en situaciones de heladas.

Los resultados obtenidos de los análisis de redes permiten conocer el tiempo necesario para llegar desde cada portal a los servicios ciudadanos primarios, o la población a la que cubre cada servicio según unos intervalos de tiempo.

En Matadepera el desplazamiento a pie desde una vivienda hasta los servicios básicos es superior a los 15 minutos en más del 50\% de los casos (farmacias, comercio de alimentación y el punto de encuentro en caso de emergencia), y en algunos hasta casi del 70\% (CAP). En cambio, son menos las viviendas que quedan a más de 15 minutos de las escuelas (41\%) y de las paradas de autobús (12\%). Por debajo de los 5 minutos, el $75 \%$ de los domicilios no tienen acceso a servicios como las farmacias, el CAP, las escuelas, los comercios de alimentación y el punto de evacuación en caso de emergencia.

La utilización de vehículo privado motorizado reduce considerablemente estas cifras, de manera que con su uso más del $90 \%$ de las viviendas están a menos de 10 minutos de todos los servicios, a excepción de la estación de bomberos (89\%). Además, más del 50\% de los domicilios, incluso en algunos casos hasta el 75\% (por ejemplo, el punto de evacuación en caso de emergencia), están a menos de 5 minutos en coche de la mayoría de servicios, a excepción de nuevo de los bomberos (40,6\%) (figura 4).

Las áreas de servicio de los diferentes servicios básicos de Matadepera muestran una situación muy similar casi en todos los casos (figuras 5 y 6). La población del núcleo urbano (sur del municipio) queda abastecida por todos los servicios en un tiempo razonable, menos de 5 minutos en los desplazamientos 
Figura 4. Tiempo de desplazamiento origen (portales) destino (servicios primarios)

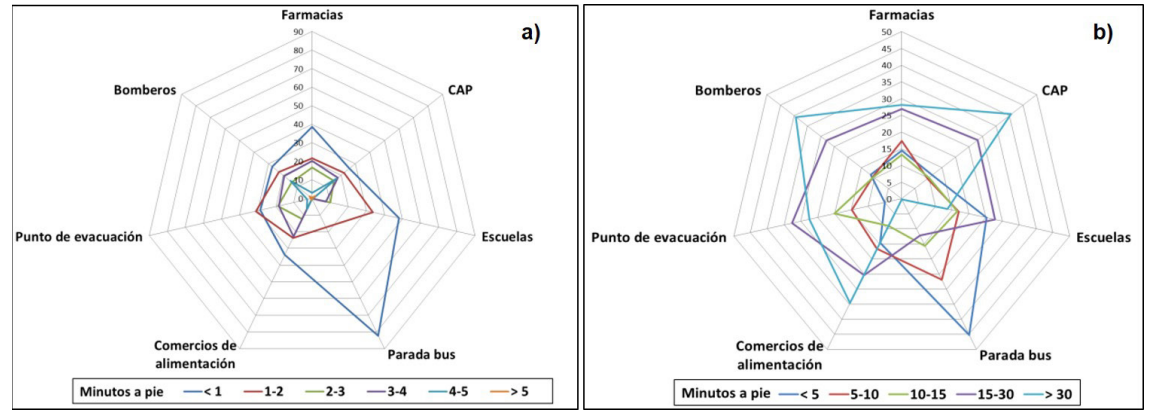

a) Resultados matriz OD des de los portales a los distintos servicios a pie; b) Resultados matriz OD des de los portales a los distintos servicios en vehículo privado motorizado

Fuente: elaboración propia.

con vehículo privado motorizado y menos de 10 a pie. Pero Matadepera se caracteriza por tener un poblamiento disperso, por lo que este análisis se ha extendido más allá del núcleo central de población. Desde estas urbanizaciones se puede llegar a cualquier servicio en menos de 10 minutos e incluso en algunos casos, como las paradas de autobuses, toda la población puede acceder en un máximo de 5 minutos en coche. Las escuelas cubren bien la zona este del municipio (urbanización Cavall Bernat y el Pla de Sant Llorenç), pero no tanto la zona oeste (Les Pedritxes y Els Rourets). Urbanizaciones como la Plana, Can Solà del Racó y Can Prat, al ser contiguas al núcleo de población, se benefician de los servicios. Si se analizan las áreas de servicio sin vehículo privado motorizado, el esquema es el mismo que el anterior, pero incrementando el coste de tiempo hasta los 30 minutos en las zonas de urbanizaciones más aisladas, hasta 10-15 minutos en el núcleo urbano y hasta 20-25 en aquellas urbanizaciones cercanas al núcleo urbano. Por lo tanto, según el estudio de Marquet y Miralles-Guasch (2014b), a excepción de la población del núcleo urbano, los habitantes de Matadepera no tienen una relación de proximidad a sus servicios básicos ya que tienen que destinar más de 10 minutos a pie para acceder a ellos.

La distribución espacial por grupo de edades de la población es un indicador que permite identificar aquellos sectores más vulnerables desde el punto de vista de la movilidad: niños, adolescentes o mayores de 70 años (figura 7). Referente al grupo de población determinado como niños (0 a 10 años), se observa que principalmente se concentran (con más de 100 niños) en la urbanización de Les Pedritxes (noreste), en la de Cavall Bernat (noroeste) y en el casco antiguo del núcleo de la población. Excepto en el casco antiguo, el coste temporal del desplazamiento a pie de los niños es superior a los 30 minutos para acceder a las escuelas. Algunos barrios, como el Pla de Sant Llorenç (noreste) o Can Torrella (centro), tienen una situación muy similar en cuanto a la movilidad de la población menor de 10 años, pero el coste temporal de 
Figura 5. Ejemplos de área de servicio (a pie)

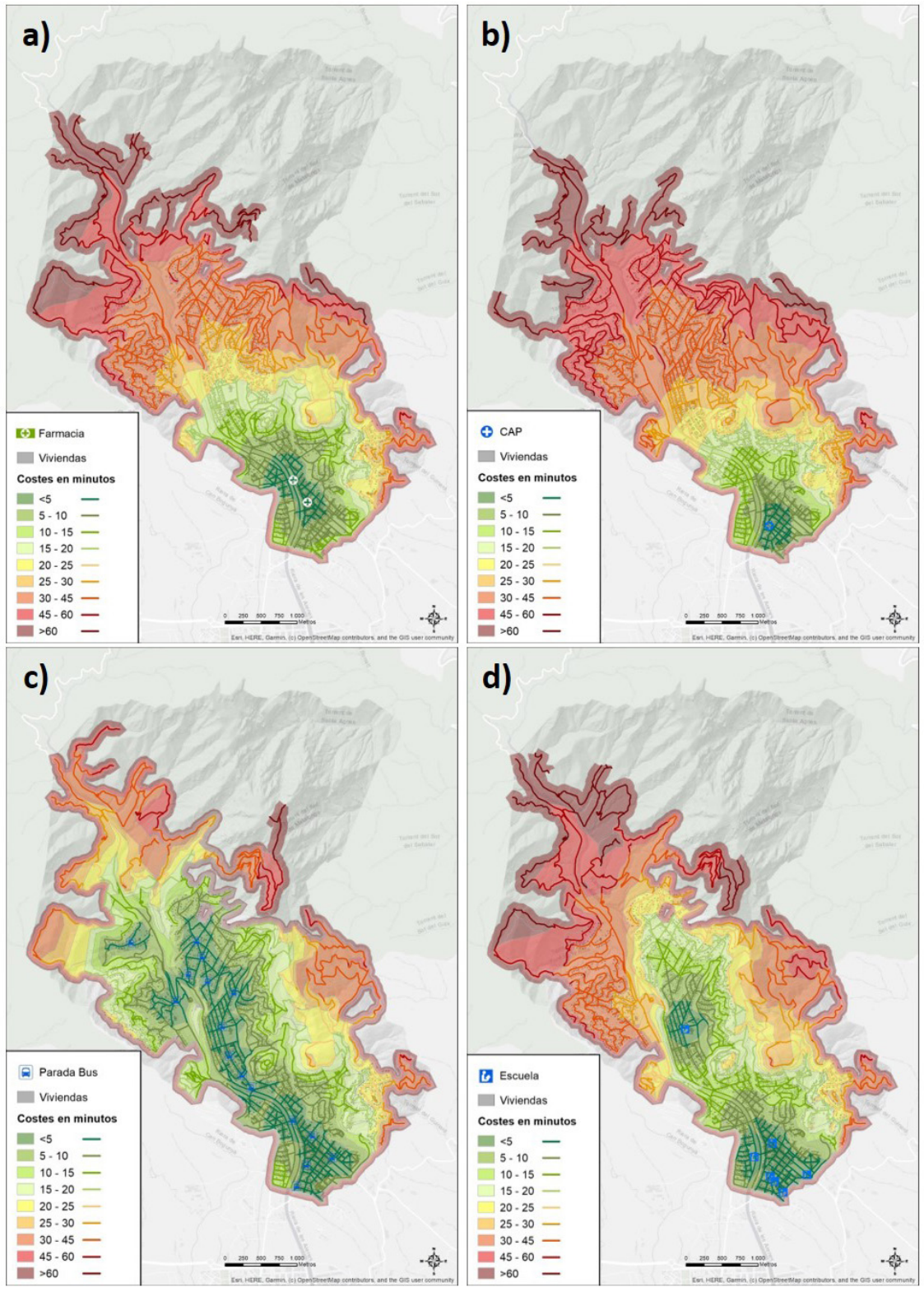

Áreas de influencia desde: a) las farmacias, b) centros de atención primaria, c) escuelas y d) paradas de autobús (a pie)

Fuente: elaboración propia. 
Figura 6. Ejemplos de área de servicio (vehículo privado motorizado)
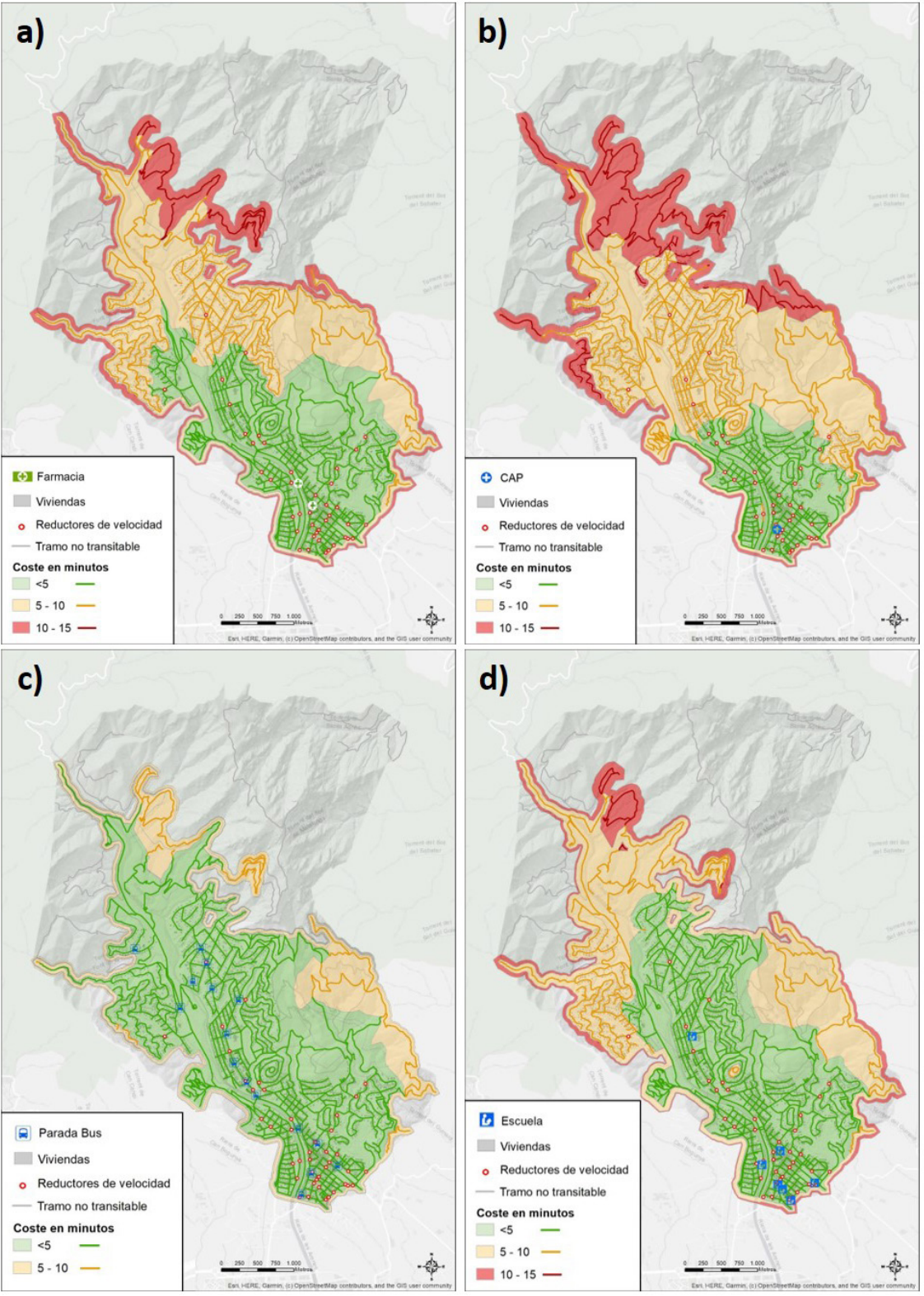

Áreas de influencia des de: a) las farmacias, b) centros de atención primaria, c) escuelas y d) paradas de autobús (vehículo privado motorizado)

Fuente: elaboración propia. 
Figura 7. Población por barrios según edad
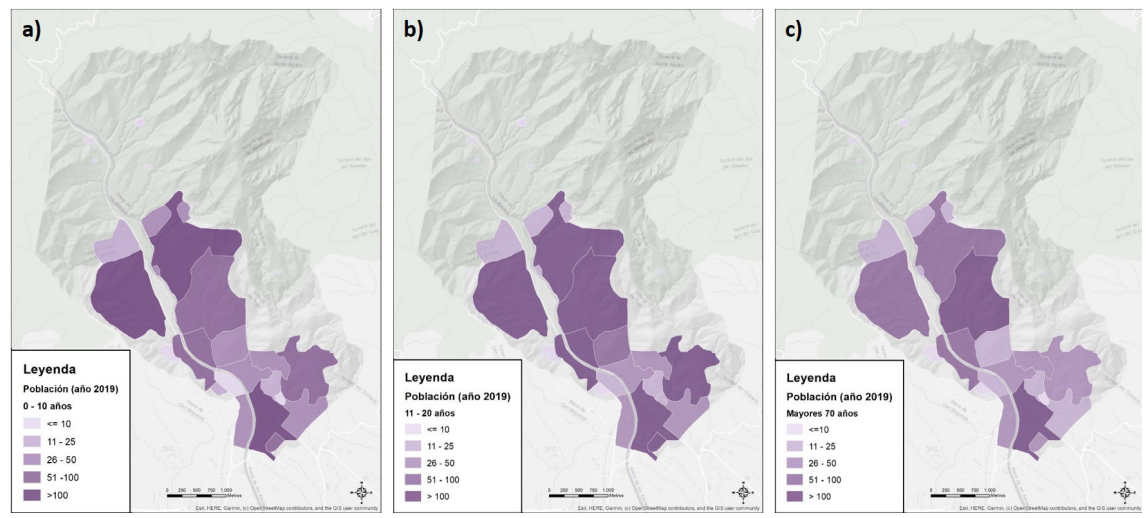

a) población de 0 a 10 años, b) población de 11 a 20 años y c) población mayor de 70 años Fuente: elaboración propia.

desplazamiento a pie disminuye a 20-25 minutos. Además, en caso de emergencia, esta población, que no tiene acceso a vehículo privado motorizado, se encuentra a más de 30 minutos a pie del punto de evacuación. Por lo tanto, en estas áreas, la población menor de 10 años tiene una dependencia total de otra persona con vehículo privado motorizado para desplazarse y reducir el tiempo de recorrido hasta la escuela a 5-10 minutos. En el grupo de edad establecido como adolescentes (11 a 20 años), el perfil es muy similar al de los niños, pero con la problemática de que barrios como el Pla de Sant Llorenç, Can Torrella o Can Solà del Racó pasan a tener más de 100 habitantes de este grupo de edad. En este caso, son muchos los adolescentes mayores de 15 años que se desplazan en moto o en cochecito a la escuela (figura 8). En cuanto al último grupo de edad, el de la población mayor de 70 años, este se concentra especialmente (con más de 100 habitantes) en el casco antiguo y en el Pla de Sant Llorenç, y entre 50-100, en Les Pedritxes, Can Torrella y Cavall Bernat.

Figura 8. Motocicletas y cochecitos aparcados al lado del instituto

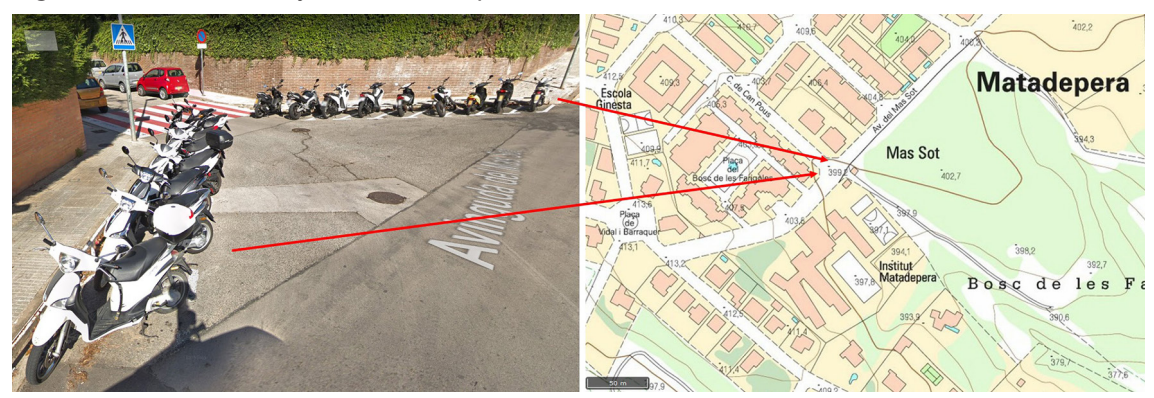

Fuente: a) Google Street View y b) topográfico del ICGC 2019. 
Esta población necesita tener acceso a diferentes servicios básicos a pie con cierta facilidad. Pero al analizar los resultados se puede ver que en la mayoría de los casos tardan más de 20 minutos a pie, o mucho más en el caso de las urbanizaciones más alejadas del centro (Les Pedritxes y Cavall Bernat), para acceder a una farmacia, a comercios de alimentación, al CAP o para desplazarse hasta el punto de evacuación en caso de emergencia.

En resumen, pues, Matadepera es una población donde el 50\% o más de sus desplazamientos no motorizados tienen un coste temporal superior a 10 minutos, con lo que supera la media de la Región Metropolitana de Barcelona $(38,6 \%)$ (IERMB, 2006). Otra característica que se desprende de dicho estudio son las distancias dispuestas a recorrer por niños y adolescentes para ir a la escuela (niños: máximo, 1.250 metros en zonas urbanas, y adolescentes: máximo, 1.550 metros en zonas urbanas) (Rodríguez-López et al., 2017). En este caso, Matadepera vuelve a sobrepasar estos valores, ya que sus niños y adolescentes, a excepción de los que viven en el casco antiguo, tienen que recorrer de 1.200 metros a 1.800 metros para ir a la escuela (el cálculo de distancia se considera de un metro por segundo, según estudios de la Generalitat de Catalunya) (Generalitat de Catalunya, n. d.).

\section{Conclusiones}

Las aportaciones de este artículo tienen una doble vertiente. Por un lado, metodológica, al aplicar el análisis de redes (matriz OD y áreas de servicio) al cálculo del coste temporal de la movilidad en zonas urbanas de baja densidad, del que no existen precedentes. Por el otro, los mismos resultados son una herramienta valiosa para la toma de decisiones sobre la gestión de la movilidad en municipios con una estructura urbana dispersa.

El trabajo realizado ha dado respuesta a las preguntas planteadas inicialmente acerca del coste en tiempo de cada portal hasta un servicio determinado, y el área de influencia en tiempo a partir de un servicio determinado. El artículo ha puesto de manifiesto los elevados costes temporales, en términos de la movilidad cotidiana, que comporta a los ciudadanos de Matadepera la actual estructura urbana del municipio. La distancia temporal a los servicios básicos de la mayoría de las urbanizaciones, la facilidad de estacionamiento cerca de los servicios básicos y la ausencia de problemas relacionados con la congestión de tráfico convierten el vehículo privado motorizado como el único medio de transporte viable para la casi totalidad de los habitantes. Esto comporta unos costes sociales, económicos y ambientales que hacen que la movilidad interna no sea sostenible. Planificar la localización espacial de algunos servicios básicos es complejo en zonas de urbanización dispersa, porque para que los equipamientos sean sostenibles y eficientes tienen que estar más cerca de los usuarios, y aquí las distancias son mucho mayores que en una ciudad compacta. Desplazarse en autobús sería una alternativa viable si se atiende a los resultados de la matriz OD, ya que la mayoría de los habitantes tienen una parada a menos de cinco minutos a pie. Sin embargo, la frecuencia de 
paso hace que esta opción quede automáticamente desestimada por parte de la mayoría de la población.

Para mitigar los aspectos negativos de la movilidad, las políticas llevadas a cabo desde la Administración deben asumir y entender el papel que juega la estructura urbana en el desplazamiento de la población a servicios como el médico, comercios, escuelas, etcétera, y el coste de abastecer a la población de otros servicios como bomberos, policía o recogida de basura.

Este tipo de estudios se convierten en una herramienta clave para la planificación urbana y la toma de decisiones en el municipio. En un futuro, la investigación se centrará en las alternativas de desplazamiento más sostenibles. No existen estudios que relacionen la velocidad en bicicleta según la pendiente, por lo que esta es una de las líneas futuras de investigación. El Ajuntament de Matadepera, conjuntamente con las escuelas del municipio y con el apoyo de la Diputació de Barcelona, ha iniciado el proyecto Camino Escolar, una iniciativa que pretende favorecer los desplazamientos sostenibles, preferentemente a pie, seguros, agradables y saludables, entre los niños, niñas y jóvenes desde su casa a la escuela, para potenciar su autonomía personal, la responsabilidad y el civismo de todos los ciudadanos. Un gran reto teniendo en cuenta el coste temporal de la distancia de toda la zona urbana del municipio.

\section{Referencias bibliográficas}

ARCGIS (n. d.). «¿Qué es la extensión ArcGIS Network Analyst?». Recuperado de $<$ https://desktop.arcgis.com/es/arcmap/latest/extensions/network-analyst/whatis-network-analyst-.htm>. [Actualizado en noviembre de 2019].

- (n. d.). «Información general sobre el trabajo con datasets de entidades». Recuperado de <http://desktop.arcgis.com/es/arcmap/10.3/manage-data/feature-datasets/ an-overview-of-working-with-feature-datasets.htm>. [Actualizado en mayo de 2020].

Ajuntament de Matadepera (n. d.). Àrea de Secretaria Padró i Estadística.

BADIA, Anna y VALLDEPERAS, Natàlia (2015). «El valor histórico y estético del paisaje: claves para entender la vulnerabilidad de la interfaz urbano-forestal frente a los incendios». Scripta Nova. Revista Electrónica de Geografía y Ciencias Sociales, XIX (521).

BANZO, Mayté (2005). «Del espacio al modo de vida: la cuestión periurbana en Europa Occidental: los casos de Francia y España (The peri-urban question in Western Europe: the cases of France and Spain)». En: ÁvILA, Héctor (coord.). Lo urbano rural: ¿nuevas expresiones territoriales?, 207-240. Cuernavaca: CRIM-UNAM.

BRUECKNER, Jan K. y FANSLER, David A. (1983). «The economics of urban sprawl: Theory and evidence on the spatial sizes of cities». The Review of Economics and Statistics, 65 (3), 479-482. $<$ http://dx.doi.org/10.2307/1924193>

BruegmanN, Robert (2005). Sprawl: A compact history. Chicago: University of Chicago Press.

Cebollada, Àngel; Badia, Anna y Vera, Ana (2020). «Movilidad Cotidiana y Cambio Modal en Zonas Urbanas de Baja Densidad. Estudio de Caso en la 
Región Metropolitana de Barcelona». Revista de Estudios Andaluces (REA), 39, 94-113. <https://doi.org/10.12795/rea.2020.i39.05>

Cussó, Xavier; Garrabou, Ramon y Tello, Enric (2005). «Social metabolism in an agrarian region of Catalonia (Spain) in 1860-1870: Flows, energy balance and land use». Ecological Economics, 58, 49-65.

<http://dx.doi.org/10.1016/j.ecolecon.2005.05.026>

Diputació de Barcelona. (n. d.). Sistema d'Informació Territorial de la Diputació de Barcelona (Sitmun). <http://sitmun.diba.cat/sitmun2/inicio.jsp>. [Actualizado en mayo de 2020].

Domene, Elena y SAURí, David (2006). «Urbanisation and water consumption: influencing factors in the Metropolitan Region of Barcelona». Urban Studies, 43 (9), 1605-1623. <http://dx.doi.org/10.1080/00420980600749969>

EEA (2006). Urban sprawl in Europe - The ignored challenge. European Environment Agency. EEA, report 10.

- (2016). Urban sprawl in Europe. European Environment Agency. Joint EEAFOEN, report 11.

Eigenbrod, Felix; Bell, V. A.; Davies, H. N.; Heinemeyer, Andreas; Armsworth, Paul R. y GASTON, Kevin J. (2011). "The impact of projected increases in urbanization on ecosystem services». Proceedings of the Royal Society of London B: Biological Sciences, 278, 3.201-3.208. <http://dx.doi.org/10.1098/rspb.2010.2754>

Ford, Alistair C.; BArR, Stuart L.; DAwsOn, Richard J. y James, Philip (2015). «Transport Accessibility Analysis Using GIS: Assessing Sustainable Transport in London». ISPRS International Journal of Geo-Information, 4 (1), 124-149. <http://dx.doi.org/10.3390/ijgi4010124>

Galster, George; Hanson, Royce; Ratcliffe, Michael R.; Wolman, Harold; Coleman, Stephan y FreiHage, Jason (2001). «Wrestling sprawl to the ground: Defining and measuring an elusive concept». Housing Policy Debate, 12 (4), 681-717. <https://doi.org/10.1080/10511482.2001.9521426>

GARDNER, Benjamin y ABRAHAM, Charles (2007). "What driver car use? A grounded theory analysis of commuters' reasons for diving». Transportation Research Part F: Traffic Psychology Behaviour, 10 (3), 187-200.

GebeyeHU, Mintesnot y TAKANO, Shin-ei (2008). «Demand Responsive Route Design: GIS Application to Link Downtowns with Expansion Areas». Journal of Public Transportation, 11 (1), 43-62. <http://dx.doi.org/10.5038/2375-0901.11.1.3>

Generalitat de CATAlunya (n. d.). «Mobilitat». <http://mobilitat.gencat.cat/ $\mathrm{ca} /$ serveis/mitjans_de_transport/a_peu/index.html $>$. [Actualizado en mayo de 2020]

GonZÁLEZ, María Jesús (2017). «Planning, urban sprawl and spatial thinking». European Journal of Geography, 8 (1), 32-43.

GUIRADO, María (2005). Fragmentation and human disturbances in prei-urban forests: effects on vascular flora (tesis doctoral). Universitat Autònoma de Barcelona.

HASSE, John E. y LATHROP, Richard G. (2003). «Land resource impact indicators of urban sprawl». Applied Geography, 23 (2-3), 159-175.

<http://dx.doi.org/10.1016/j.apgeog.2003.08.002> 
Honnay, Oliver; Endels, P.; Vereecken, H. y Hermy, Martin (1999). "The role of patch area and habitat diversity in explaining native plant species richness in disturbed suburban forest patches in northern Belgium». Diversity and Distributions, 5, 129-141. <https://doi.org/10.1046/j.1472-4642.1999.00047.x>

Hortas-Rico, Miriam y Solé-Ollé, Albert (2010). «Does Urban Sprawl Increase the Costs of Providing Local Public Services? Evidence from Spanish Municipalities». Urban Studies, 47 (7), 1.513-1.540. <http://dx.doi.org/10.1177/0042098009353620>

ICGC (n. d.). Institut Cartogràfic i Geològic de Catalunya. Datos LiDAR. <http://www. icgc.cat/es/Descargas/Elevaciones/Datos-lidar>. [Actualizado en mayo de 2020].

IDESCAT (n. d.). Institut d'Estadistica de Catalunya. <https://www.idescat.cat/>. [Actualizado en mayo de 2020].

IERMB (2006). Enquesta de Mobilitat Quotidiana. Institut d'Estudis Regionals i Metropolitans.

IGN (n. d.). Instituto Geográfico Nacional. <https://www.ign.es/web/ign/portal>. [Actualizado en mayo de 2020].

IndOVINA, Francesco (1990). La città diffusa. Venecia: Istituto Universitario di Architettura di Venezia. Dipartamento di Analisi Economica e Sociale del Territorio.

Lambin, Eric F.; Turner, B. L.; Geist, H. J.; Agbola, S. B.; Angelsen, A.; Bruce, J. W.; Coomes, O. T.; Dirzo, R.; Fischer, G.; Folke, C.; George, P. S.; Homewood, K.; Imbernon, I.; LeEMAns, R.; Li, X.; Moran, E. F.; Mortimore, M.; Ramakrishnan, P. S.; Richards, J. F.; Skanes, H.; Steffen, W.; Stone, G. D.; Svedin, U.; VeldKamp, T. A.; Vogel, C. y Xu, Jianchu (2001). «The causes of land-use and land-cover change: moving beyond the myths». Global Environmental Change, 11 (4), 261-269.

<http://dx.doi.org/10.1016/S0959-3780(01)00007-3>

LOWRY, Ira S. (1988). «Planning for Urban Sprawl». Special Report 220. Looking Ahead, the Year 2020, 275-312. Washington: Transportation Research Board, National Research Council.

MARCH, Hug y SAURí, David (2010). «The suburbanization of water scarcity in the Barcelona metropolitan region: Sociodemographic and urban changes influencing domestic water consumption». The Professional Geographer, 62 (1), 32-45. <http://dx.doi.org/10.1080/00330120903375860>

Marquet, Oriol y Miralles-Guasch, Carme (2014a). "The walkable city and the importance of the proximity environments for Barcelona's everyday mobility». Cities, 42 (B), 153-280. <https://doi.org/10.1016/j.cities.2014.10.012>

- (2014b). "Walking short distance. The socioeconomic drivers for the use of the proximity in everyday mobility in Barcelona». Transportation Research Part A: Police and Practice, 70, 210-222. <https://doi.org/10.1016/j.tra.2014.10.007>

MARTínEZ-AlIER, Joan y RoCA, Jordi (2001). Economia ecológica y política ambiental. México DF: Fondo de Cultura Económica.

Miralles-Guasch, Carme; Martínez Melo, Montserrat y Marquet, Oriol (2014). «On user perception of private transport in Barcelona Metropolitan area: an experience in an academic suburban space». Journal of Transport Geography, 36, 24-31.

MUC (n. d.). Mapa Urbanistic de Catalunya. Recuperado de <http://territori.gencat. cat/ca/06_territori_i_urbanisme/observatori_territori/mapa_urbanistic_de_catalunya/>. [Actualizado en mayo de 2020]. 
MuÑIz, Iván y GARCÍA-LÓPEZ, Miquel-Àngel (2013). "Anatomía de la dispersión urbana en Barcelona». EURE, 39 (116), 189-219.

MuÑOZ, Francesc (2003). «Lock living: urban sprawl in Mediterranean cities». Cities, $20(6), 381-385$.

ONU (2018). World Urbanization Prospects 2018. Department of Economic and Social Affairs. ONU. <https://population.un.org/wup/>. [Actualizado en mayo de 2020].

OrDEN VIV/561/2010, de 1 de febrero. Boletín Oficial del Estado. Jueves, 11 de marzo de 2010.

PÁez, Antonio; Mercado, Ruben; Morency, Catherine; Roorda, Mathew J. y FarBER, Steven (2011). «Distance traveled in three Canadian cities: Spatial analysis from the perspective of vulnerable population segments». Journal of Transport Geography, 19 (1), 39-50. <https://doi.org/10.1016/j.jtrangeo.2009.09.013>

Palma, André y Rochat, Denis (2000). «Mode choices for trips to work in Geneva: an empirical analysis». Journal of Transport Geography, 8 (1), 43-51. <https://doi.org/10.1016/S0966-6923(99)00026-5>

PALOMINO, David y CARRASCAL, Luis M. (2006). «Urban influence on birds at a regional scale: A case study with the avifauna of northern Madrid province». Landscape and Urban Planning, 77, 276-290.

Pérez, N.; Pérez, M.; Ruiz, N. y Coll, F. (2017). «Relación entre forma urbana y patrones de movilidad: el caso del área metropolitana de Barcelona». Comercio Internacional y Empleo: una perspectiva regional. XLIII Reunión de Estudios Regionales-XIII. Congreso de Ciencia Regional de Andalucía. International Conference on Regional Science. Sevilla: Asociación Española y Andaluza de Ciencia Regional.

Radeloff, Volker C.; Hammer, Roger B. y Stewart, Susan I. (2005). «Rural and suburban sprawl in the U. S. Midwest from 1940 to 2000 and its relation to forest fragmentation». Conservation Biology, 19 (3), 793-805.

Rodríguez-López, Carlos; Salas, Zeus; Villa-González, Emilio; Borges-Cosic, Milkana; Herrador-Colmenero, Manuel; Medina-Casaubón, Jesús; Ortega, Francisco B. y Chillón, Palma (2017). «The threshold distance associated with walking from home to school». Health Education \& Behavior, 44 (6), 857-866. <http://dx.doi.org/10.1177/1090198116688429>

Sede Electrónica del Catastro (n. d.). Ministerio de Hacienda, Gobierno de España. Recuperado de <https://www.sedecatastro.gob.es/>. [Actualizado en mayo de 2020].

Salvati, Luca; Sateriano, Adele y Bajocco, Sofia (2013). «To grow or to sprawl? Land cover relationship in a mediterranean city region and implications for land use management». Cities, 30, 113-121. <https://doi.org/10.1016/j.cities.2012.01.007>

SAmOnA, Giuseppe (1968). Piano urbanistico del Trentino. Venecia: Marsilio Editore.

SCHNEIDER, Annemarie y WoOdCOCK, Curtis. E. (2008). "Compact, dispersed, fragmented, extensive? A comparison of urban growth in twenty-five global cities using remotely sensed data, pattern metrics and census information». Urban Studies, 45, 659-692. <https://doi.org/10.1177/0042098007087340>

Sevtsuk, Andres y MeKonnen, Michael (2012). «Urban Network Analysis. A new toolbox for ArcGIS». Revue Internationale de Géomatique, 22 (2), 287-305. <https://doi.org/10.3166/rig.22.287-305> 
SeVtSuK, Andres; Kalvo, Raul y EKMEKCI, Onur (2016). «Pedestrian accessibility in grid layouts: the role of block, plot and street dimensions». Urban Morphology, 20 (2), 89-106.

SITMUN (n. d.). Sistema d'Informació Territorial Municipal. <http://sitmun.diba.cat/ sitmun2/inicio.jsp?lang=ca>. [Actualizado en mayo de 2020].

Travissi, Chiara M.; CAmagni, Roberto y NijKamp, Peter (2010). «Impacts of urban sprawl and commuting: a modelling study for Italy». Journal of Transport Geography, 18 (3), 382-392.

<http://dx.doi.org/10.1016/j.jtrangeo.2009.08.008>

Turbé, Anne; Toni, Arianna de; Benito, Patricia; Lavelle, Perrine; RuIZ, Nuria, Putten, Wim H. van der; Labouze, Eric y Mudgal, Shailendra (2010). «Soil biodiversity: functions, threats and tools for policy makers». Bio Intelligence Service, 248 p. París: Bio Intelligence Service, IRD y NIOO. Recuperado de <http:// ec.europa.eu/environment/soil/biodiversity.htm>. [Actualizado en mayo de 2020].

Zhou, Yang; FAnG, Zhixiang; Thill, Jean-Claude; LI, Qingquan y LI, Yuguang (2015). «Functionally critical locations in an urban transportation network: Identification and space-time analysis using taxi trajectories». Computers, Environment and Urban Systems, 52, 34-47.

$<$ http://dx.doi.org/10.1016/j.compenvurbsys.2015.03.001> 
